\title{
A Platform for Multi-Messenger Observing
}

\author{
John Lightfoot ${ }^{1}$ \\ UKATC \\ Blackford Hill, Edinburgh, U.K. \\ E-mail: john.lightfootestfC.ac.uk \\ Josep Colomé \\ IEEC \\ 08034 Barcelona, Spain \\ E-mail: colomedieec.cat
}

\section{Mark Kettenis}

JIVE

7991 PD Dwingeloo, The Netherlands

E-mail: kettenisejive.eu

\section{Dave Morris}

Edinburgh University

Blackford Hill, Edinburgh, U.K.

E-mail: dmreroe.ac.uk

\section{Marjan Timmer}

ASTRON

7991 PD Dwingeloo, The Netherlands

E-mail: timmerlastron.nI

This paper reviews how multi-facility observations are coordinated today and concludes that the process will be insufficient for the flood of transient follow-up campaigns expected in the era of gravity wave, cosmic ray and neutrino detectors, and the LSST. Improvement is also required in the coordinated observation of steady sources, and in the optimization of synchronized surveys.

Study of the efforts currently underway to resolve the problems shows much good work but a lack of overall coordination. We suggest that, even without a master plan, the various separate components that have been built, plus new ones not yet considered, could be assembled to make a web 'platform' that would be a useful aid to coordinated observing in the future.

A pilot use-case is used to develop a plan for that platform and a simplified prototype has been constructed.

The New Era of Multi-Messenger Astrophysics - Asterics2019

25 - 29 March, 2019

Groningen, The Netherlands

\section{${ }^{1}$ Speaker}

(c) Copyright owned by the author(s) under the terms of the Creative Commons 


\section{The Observing Process}

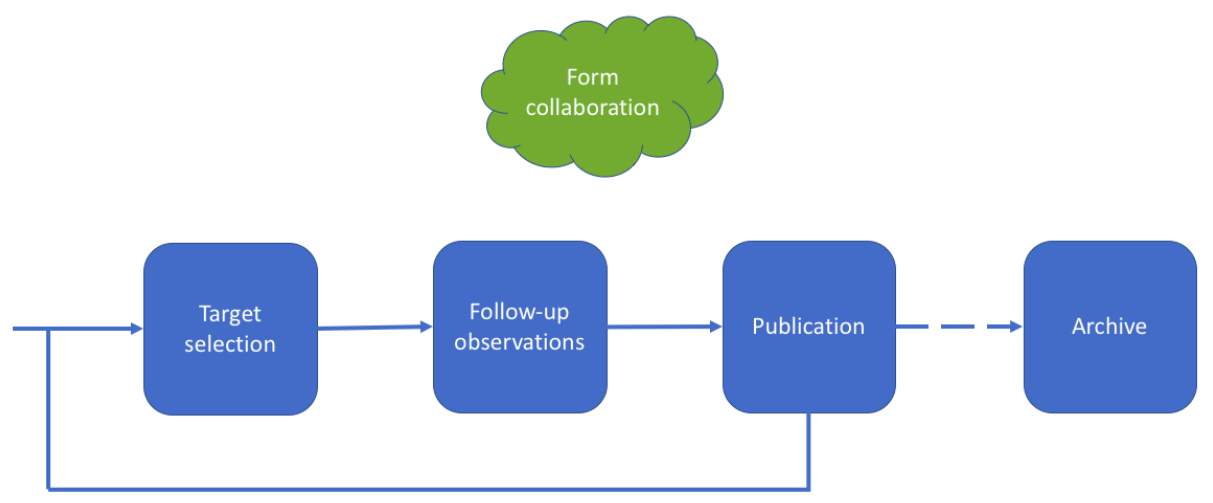

Figure 1. The follow-up observing process.

Elements of the typical process for multi-messenger (MM) or multi-wavelength observing are illustrated in Fig.1. The collaboration is an important component, whose formation behind the scenes is not normally considered.

This informal process has worked well but its low efficiency is challenged today by gravity wave and neutrino telescopes that have good sensitivity but poor localization. Further problems will arise with the arrival of synoptic telescopes, like the LSST and SKA, able to deliver millions of alerts per night. Simultaneous observation of steady sources is also valuable and in need of tools that promote and optimize collaboration.

An updated process, fit for the new era, must accomplish the following things:

1. Filter from the millions of potential targets per night the few that are of interest to the observer and which it is feasible to pursue.

2. Perform follow-up observations efficiently. This requires:

a. A collaboration (usually), for breadth of expertise and work capacity.

b. A place for the collaboration to communicate, share data, and construct joint observations.

c. Knowledge of the observing context - past observations and any already planned - to take advantage of serendipity and to avoid duplication.

3. Archive data as a coherent assembly with all the metadata necessary to reconstruct the story of the observing campaign at a later date.

\section{Ideas under Development}

Many efforts are underway to prepare for the coming transient flood and to facilitate simultaneous observations of targets, including:

- Event broker development. Originally developed to simply relay event streams from facilities to subscribers, these increasingly do much more. For example, Lasair [1], a prototype broker being developed in the UK for the LSST can: 
- Maintain 'watch lists' that sieve the event stream through filters tailored to an observer's interest.

- Add to each event the estimated likelihood that it is a supernova.

- For gravity wave alerts, as an aid to follow-up, display the alert skymap with catalogue galaxies superimposed and ranked in host likelihood order.

Likewise, AMON [6] provides a valuable service by focusing on alert correlation and collation. Many other brokers are available, each with its strengths, and we expect that new capabilities will continue to be added to them all.

- Construction of Target and Observation Managers (TOMs), web tools used to set up and schedule observations. The LCO is developing a toolkit of components, which groups can use to build their own bespoke TOM [2].

- Aids to the formation and work of collaborations. Two approaches have been tried so far:

- The classic way, where the collaboration comes before the telescope time. ENGRAVE [3], a group of more than 240 scientists from ESO member states, was awarded VLT 'target of opportunity' time over several semesters for gravity-wave follow-up. ENGRAVE is centrally organized and will perhaps run like a ship, with officers, people on watch, etc.

- Social networking. SmartNet [4] is a web site that any astronomer interested in collaboration can join. A member spotting a potential target starts an 'observing campaign', creating a site page where observations made or planned can be viewed, plans discussed, etc. Emails are automatically sent to members advertising the campaign, and people join by contributing data.

- Establishment of interface protocols by the IVOA e.g. ObjVisSAP, a protocol for describing target visibility and ObsLocTAP, for a schedule of observations [5].

\section{The MM Platform}

The previous section shows that much good work is being done to improve coordinated observing, but the effort is itself not coordinated. We ask, "can the components being developed separately be assembled into a collaborative 'platform' that provides a complete path from target selection, through follow-up observation planning and reduction, to archiving?" Are there any gaps that need filled for this to be possible? Such a platform would naturally provide a central 'place' for news and information exchange, and for the deployment of related tools e.g. for the optimization of coordinated multi-observatory surveys.

In the last 4 months of ASTERICS a project was set up to develop use-cases for such a collaborative 'platform', produce an initial design and implement a prototype. Space precludes a full description of the work, so here we concentrate on how the platform would handle one usecase that captures much of the required functionality:

1. Using a range of brokers to filter alert streams, building on the varied capabilities that they offer, present to the user a list of potential targets.

2. The user clicks on a target to select it for follow-up, acting alone or as a member of a collaborating group. Platform 'groups' can be private or public. Private ones, following ENGRAVE, would ask new members to sign to a publication agreement 
before joining, and have private observing pages. Clicking on a target starts its 'observing story', a table of links to observations with space for supporting notes.

3. The user clicks to add a new observation to the story and selects the instruments to be used, after which facilities are interrogated for scheduled observations and target visibility. This information is displayed and used to construct a possible schedule.

4. The potential schedule, which could involve one facility or many, is ready for execution but lacks permission to use the target facilities; this is a point of difficulty for any collaborative scheme. A possible solution would have permissions given by group members inserting the relevant 'keys' into the schedule. Members having time on a particular facility would hold its key and use it to control the pooling of their resource.

5. The schedule is then submitted for execution. Completed and reduced observations are linked-to from the observation story for viewing by the group. At any stage, discussion on the platform between group members would be stored as part of the 'story'; in a sense a richer implementation of a SmartNet 'campaign'.

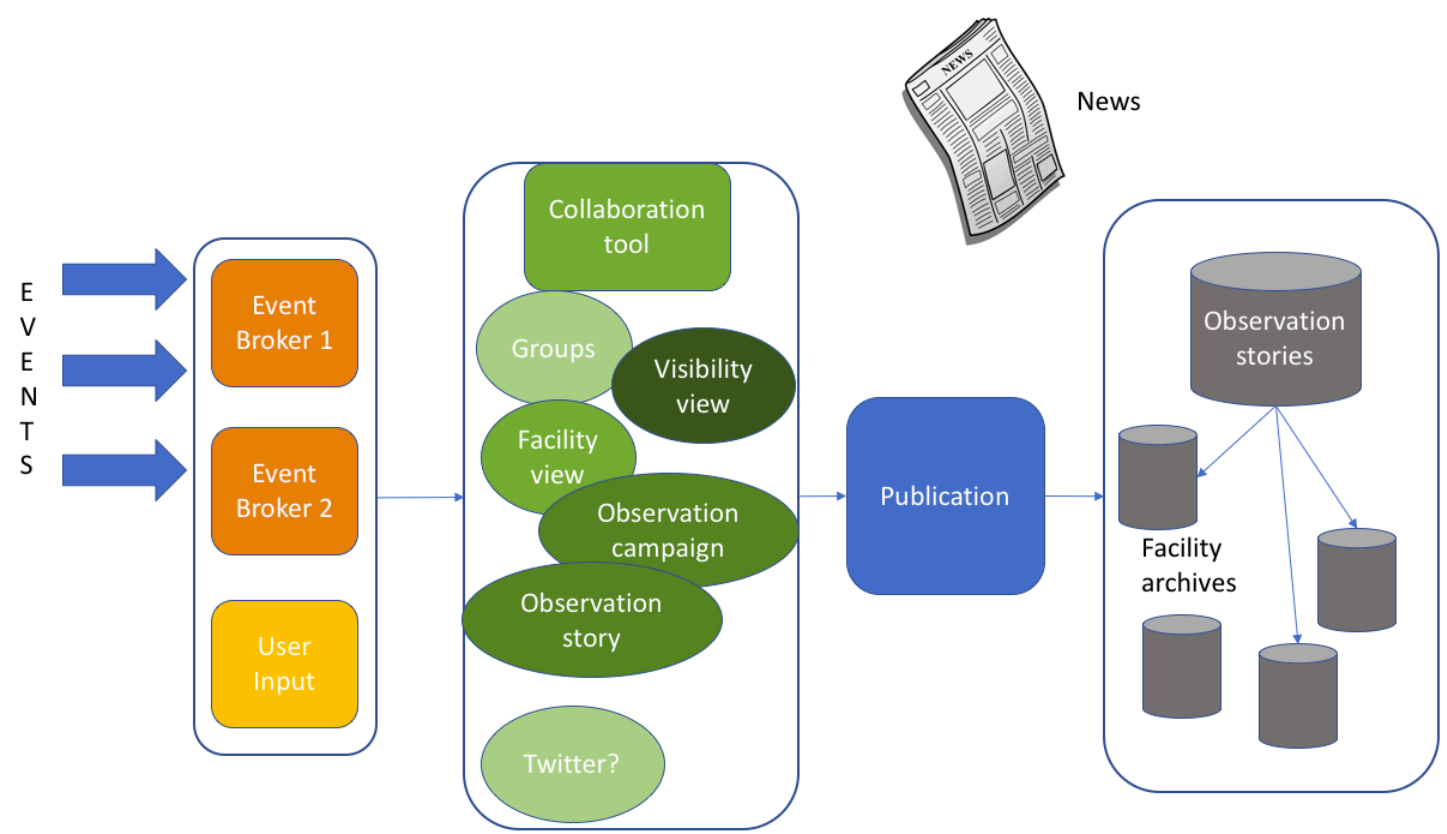

Figure 2. An outline of the components in the proposed MM platform

The components of the MM platform are shown in Fig 2, grouped into the same boxes as Fig. 1. The making of the collaboration is now implicit in the process. Note the area for 'news and views', where people can report work done in this field or comment on it; information exchange that will help coordinate further development.

\section{Acknowledgement}

This work was supported by ASTERICS, a European Commission Horizon 2020 Project, grant agreement n. 653477. 


\section{References}

[1] The Lasair broker. https://lasair.roe.ac.uk

[2] TOM Toolkit. https://lco.global/tomtoolkit/

[3] The ENGRAVE collaboration. http://www.engrave-eso.org/

[4] The SmartNet project. http://www.isdc.unige.ch/smartnet/

[5] IVOA draft protocols. http://www.ivoa.net/documents/ObjVisSAP/20180713/index.html and http://www.ivoa.net/documents/ObsLocTAP/index.html

[6] Astrophysical Multimessenger Observatory Network (AMON). https://www.amon.psu.edu/ 